\title{
A Distributed Intelligent Computer/Telephony Network Integration Architecture for Unified Media Communication
}

\author{
Plamen L. Simeonov ${ }^{+}$, Peter Hofmann* \\ + Siemens AG: Plamen.Simeonov@sietec.de \\ *Technical University Berlin, FSP-PV/PRZ: peterh@prz.tu-berlin.de
}

\begin{abstract}
This paper presents a distributed IN archit ecture called @INGate [1] [2], that elaborates a Unified Media Communications Service (UMCS) providing both messaging and telephony services between circuit switching and packet networks. It represents an InterWorking Unit (IWU) in CTI networks allowing the user access to a Unified Message Store-Line (UMSL) via traditional PSTN/ISDN equipment such as telephones and fax machines on the one side and networked computers equipped with mail readers and Web browsers on the other side to enable both online and off-line communication.
\end{abstract}

The core of this new architecture is the NetworkBridge Service Node (NB-SN), a distributed intelligent network (IN) element consisting of a Channel Matrix Switch (CMS) to connect to the PSTN/ISDN, several Ressource Platforms (RP) containing Media Conversion Processors (MCP) to perform the media translation in the required interchange formats, an Internet Gateway (IG) to hold the subscribers' mailboxes and provide the Internet connectivity, and a Service Node Controller (SNC) to manage the overall service logic and the above NB-SN components. The Service Node itself is managed along with other nodes in a distributed IN by a TMN-compliant Operation, Administration and Maintenance (OAM) center.

The @INGate system was jointly developed by Siemens AG and the Technical University Berlin.

Keywords: CTI, IWU, PSTN/Internet-Gateway

I. Introduction

The @INGate project designed an InterWorking
Unit (IWU) between ISDN/PSTN (TE2) and Internet Terminals (IT), cf. fig. 1, [3], which hosts a generic Unified Media Communication Service (UMCS). This architecture addresses three basic elements:

-protected network links: Channel Matrix

Switch (CMS) facing PSTN/ISDN (incl. connections to mobile radio networks) and Internet Gateway (IG);

-enhanced media contents: Resource Platforms (RP) using Media Conversion Processors (MCP);

-reliable service and resource control: Service Node Controller (SNC).

\section{II. @INGate Reference Model}

The @INGate Reference Model contains the following network elements (cf. fig. 2):

-Network-Bridge Service Node (NB-SN)

-Service Data Server (SDS)

- Operation, Administration and Maintenance

Center (OAMC)

-WWW Server

-Firewall

The Network-Bridge Service Node (NB-SN) consists of the following modules:

-Service Node Controller (SNC)

-Resource Platforms (RP)

- Media Conversion Processors (MCP)

-Channel Matrix Switch (CMS)

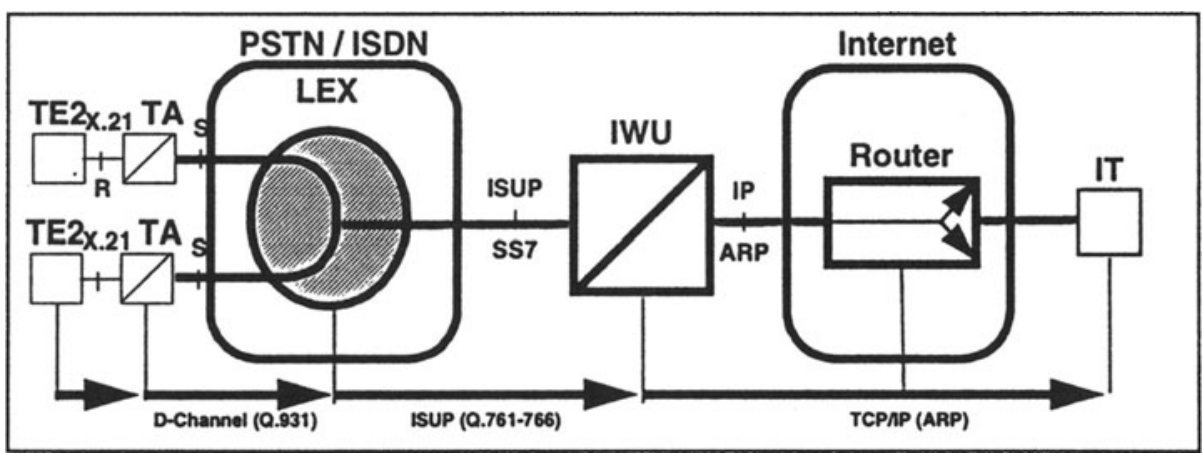

Fig. I @INGate as IWU

Intelligent Networks and Intelligence in Networks D. Gaiti (Ed.) Published by Chapman \& Hall 1997 IFIP 


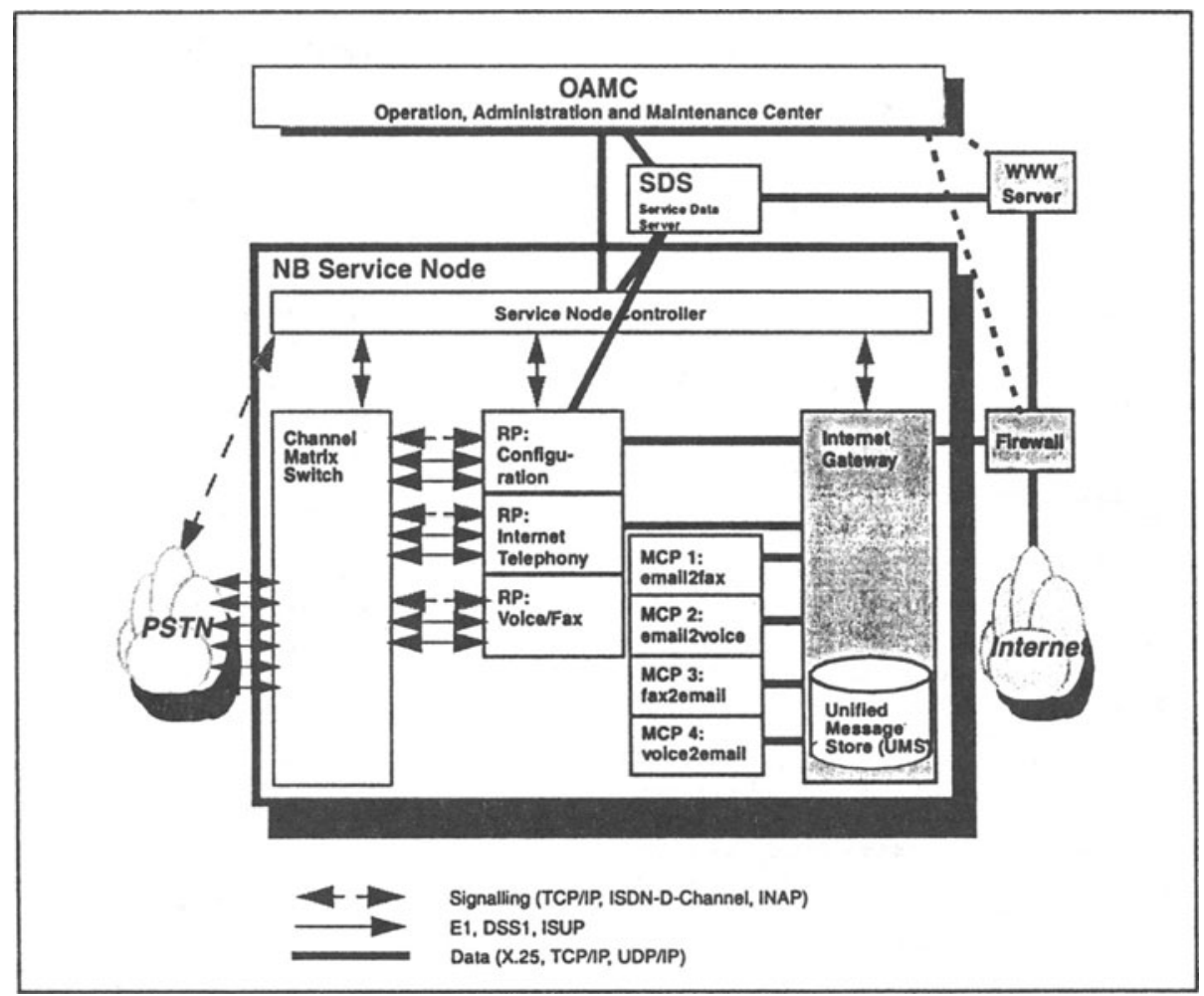

Fig. 2 @INGate Reference Model

\section{-Internet Gateway (IG)}

The Service Date Server (SDS) holds all serviceand subscriber relevant information such as configuration data, user profile information and Automated Message Acount (AMA) records in a database.

The OAM center allows access to all the components of the @INGate Service Node, e.g. via telnet sessions to UNIX hosts or SNMP-based management.

The WWW Server supports WWW-based management of all NB-SN components in addition to the TMN-conformant OAM workstation, as well as Customer Service Control (CSC) such as configuration of service specific parameters through the Internet by using security-enhanced Web browsers.

- Currently, the UMCS logic in the SN Controller guarantees the secure service access from the telephony network, whereas a dedicated firewall computer connects the Network-Bridge Service Node to the Internet.
1II. Network-Bridge Service Node (NB-SN)

The Network-Bridge Service Node represents the core of the Interworking Unit between PSTN/ ISDN and Internet. In the following sections we describe its components.

\section{A Service Node Controller (SNC)}

The Service Node Controller controls the allocation and deallocation of resources in the Resource Platforms and the overall play-out of the service. Services offered by the NB-SN are implemented in the SNC by Service Logic Programs (SLPs) interfacing to their subparts and resources in the RPs directly or via the matrix switch.

\section{B Resource Platform (RP)}

The Resource Platforms implement specific resource functions such as voice/fax reception, DTMF/voice recognition, storage, play of announcements, etc. 
Appropriate RPs are assigned to incoming and outgoing calls, as well as to service logic by the SNC. There are four different RP types in @INGate: one for recording/sending fax and voice messages, one for storage (mailbox, UMS), one for the interface to Internet Telephony, and one for configuration of user profiles via DTMF/VR.

\section{Media Conversion Processors (MCP)}

The Media Conversion Processors have the task to convert media messages

1. between RP-specific and Internet formats, and

2. between two different RP-specific formats.

\section{Channel Matrix Switch (CMS)}

The Channel Matrix Switch is controlled by the SNC and routes calls from the PSTN/ISDN to the appropriate resource platforms via circuit and call related signalling (CCS7 interface of ISUP).

\section{E Internet Gateway (IG)}

The Internet Gateway basically consists of a Unified Media Storage (UMS) and telephony software. The UMCS logic is located at the SNC. The software consists of a standard Internet Telephony (IT) client modified for use as a gateway, and an interface to a special ressource hardware handler. Since most IT applications today support half-duplex operation, only half-duplex communication is implemented at the moment. This coincides with the fact that the selected resource hardware (voice modem) also performs only a half-duplex voice recording and playback.

Note: The Internet Telephony Gateway logically belongs to the Internet Gateway. However, because of real-time constraints it will be located on the Ressource Platform. This may be a special Internet Telephony RP (IT-RP), if special hardware/operating systems are necessary.

\section{Unified Media Communication Service (UMCS)}

The @INGate project aims at implementing a generic Unified Media Communication Service that can be accessed from both the switching telephone network and the packet-driven Internet. This service imposes some constraints on the message format that is intended to support both worlds:

- The identity of the subscriber (i.e. owner of the message) must be verifyable.

- The unified message format must accommodate both telephony (c.g. G3 fax) and Internet data formats (e.g. JPEG, ASCII text).

-The format must handle multi-part messages that occur in the Internet world (MIME-format emails).
-The message format must identify the type of the content data it encapsulates.

-The message format must be efficiently accessible from both Internet e-mail software and telephony services residing in the RPs.

There are several choices for the implementation of such a Unified Message Format (UMF), especially if timing and performance of necessary conversion procedures are taken into account. The messages might be stored either in a proprietary format (e.g. involving a multimedia database or special file formats) or in a standard format (e.g. standard UNIX mailbox format). The proprietary format has the advantage that it can be implemented in a very efficient manner compared to ordinary UNIX mailboxes that store all data (even long audio files) in one mailbox file. The drawback is, however, that standard Internet daemons such as POP3 daemons have to be rewritten to use this new format. This would also make running a POP daemon computationally more expensive. There are two strategies to solve the conversion problem:

- "Lazy" Conversion of media types on demand, e.g. when a user accesses her mailbox using her Internet MUA and the mailbox contains voice messages enter using a telephone the POP daemon converts these voice messages into MIME audio/basic.

-All incoming messages are converted to/from a canonical format (CF) as soon as they come in. If the CF is the standard UNIX mailbox with MIME types at least for the Internet access, no conversion on-the-fly is required.

The standard UNIX mailbox with a canonical format was chosen. In a later revision the proprietary format with lazy conversion should be favoured because of better performance.

Currently, from the PSTN/ISDN side only the UMCS subscriber can reach Internet Telephony users from an analog telephone. She can then select the desired party using DTMF tones (either from a shortlist or by entering the IP number). The Internet side is not limited to subscribers: everyone can call a PSTN/ISDN number from the Internet, provided that she is willing to pay by credit card or using digital cash for using UMCS.

\section{Operation, Administration, and Maintenance (OAM)}

The OAM workstation allows access to all the components of the Network-Bridge Service Node (NB-SN). The structure of the @INGate architecture is oriented on IN Standards. TMN standards such as [5] and [6] are used to support management within the $\mathbb{N}$. The management information is processed by distributed SNMP agents inside the different NB-SN components. 
One of the main challenges of TMN integration in Intelligent Networks is the lack of usable standards. We used the ETSI Baseline documents [7] and the ITU-T Recommendations to map the TMN Functional Model to the @INGate physical architecture (fig. 3).

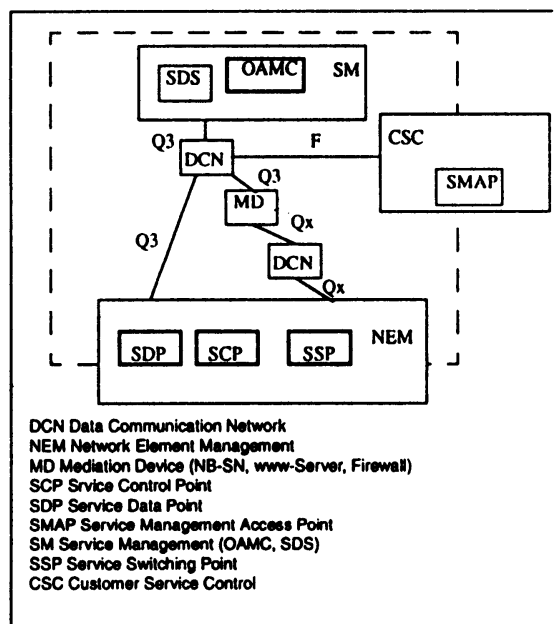

Fig. 3 Mapping of TMN to @INGate

The implementation of the management platform is based on HP OpenCall, which is used to control the IN management aspects of the service node. The Customer Service Control (CSC) management is supported over the Internet by HP OpenView as part of the OpenCall platform, with additional modules implemented by the PRZ lab at the TU Berlin. Some management information is accessible via a Web interface, mainly to support service subscribers to access and change their profiles using the Internet .

The @INGate management defines three main services:

\section{A Service \& Subscriber Administration}

This management service includes the needed functions for the administration of user profiles. The operator has total access to this data. The service subscriber can modify certain data in the user profile to get the service fitted to her needs.

\section{B Operation \& Maintenance}

This management service offers the functions for management of the service node operation. Typical functions are load-control, reachability, availability and statistics for billing and control purposes.

\section{Element Management}

This management service offers the functions needed by the SNC to control the modules inside the NB-SN. It behaves to upper TMN instances as a single Network Element, representing the Network Elements inside it.

\section{Service Data Server (SDS)}

The Service Data Server (SDS) is a common data repository for all components of the @INGate system. It holds information about

-configuration of resources

- available conversion functions

- PSTN/ISDN ports

-configuration of services

- distribution of processes

- configuration of recovery functions

-subscriber profile data

- personal data

- billing information (bank account, special

usage plans etc.)

- available conversion functions

- PSTN/ISDN port preferences (fax number

for email digests etc.)

Resource and service configuration can be accessed and manipulated by the OAM component. Subscriber profile data can also be accessed by OAM, the subscriber herself can also change parts of the profile using the Web-based interface (e.g. email-to-fax number). The Charging and Billing component uses the billing information in the subscriber profile.

The SDS holds for each subscriber a certain amount of profile information some of which can be changed by the subscriber using the Web or the telephony (DTMF) based interface. The following list gives some of the profile attributes and their description:

- User Identifier: a unique integer number for each user stored in the Subscriber Database.

-User Name: the full user name (e.g. "Erika Musterfrau").

-Login Name: a short unique user handle (e.g. "erikam");

-Account Activation Time: the time when the user account becomes valid.

-Account Deactivation Time: the time of the automatic expiration of the account.

-Billing Policy: the billing policy to be applied by the Billing Subsystem.

-Security Identifier: an identifier specific to the underlying security system.

-Security Data: information specific to the underlying security system.

-Address: the user/subscriber address used by the Billing System to send the accounts/bills. 
-Privilege Attributes: indication of the invocation privileges for the different end-user roles.

- Telephone Number: the telephone number of the user/subscriber.

-Mailing Address: the mailing address of the user/subscriber.

- Language Preference: the language preference of the user; this data can be used by the system to determine the the user interface.

-Account Balance: the current account balance of the user.

-Fax Number: the default fax number to be used for the email-to-fax service.

-Auto Fax Times: an array of time specifications; at each of these times the email-to-fax system sends all new messages to the fax number in the Fax Number field.

\section{WWW Server}

The WWW-Server serves a dual purpose in the architecture of the @INGate system:

-it allows remote WWW-based management of @INGate services as an alternative to the centralized OAM concepts.

-it allows the subscribers to modify parts of their personal profiles that are held in the SDS.

There are several NB-SN components to be managed using the WWW-Interface. Since not all of them directly support the configuration via HTML, some database conversions were implemented:

-SNMP MIBs into HTML

-database tables (especially in the SDS) into HTML

The following components can be managed with the WWW-server:

-Mail Server in the Internet Gateway (native HTML-Interface)

- Service Node Controller (MIB-to-HTML)

- Ressource Platforms (MIB to HTML)

-SDS (forms-based access to subscriber profile database: self-subscibtion, CSC)

The @INGate Service Node will distribute its services securely through a SSL-enabled [4] server (SNC, cf. fig. 2) which requires the usage of a certificate-based authentication scheme.

\section{Charging and Billing (C\&B)}

The UMC service will be accessible from both PSTN/ISDN and Internet. It should be able to handle service requests from POTS subscribers and from anonymous users originating from the Internet 18-11/.

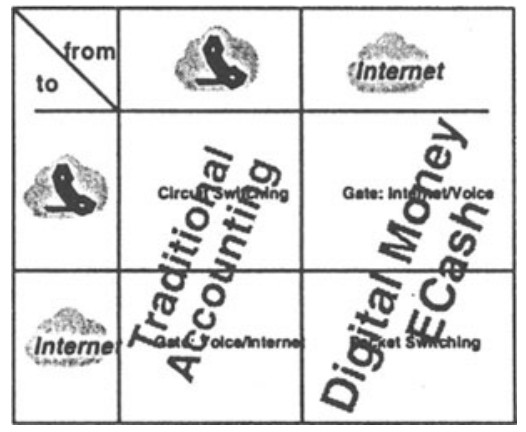

Fig. 4 Billing Areas Internet/N

\section{Related Work}

We look upon every approach of combining internet services to a telecommerce platform as related work. Regarding our activities on billing for anonymous customers the closest relationship exists to ecash shops [8], [9]. We cover the activities and movements of other billing or secure transaction initiatives like SET [10] but our main concern in @INGate is the evolution of digital money [11] which supports the distributed NB-SN architecture.

\section{Conclusions and Outlook}

This paper has shown an exciting new way to integrate two worlds that have evolved very differently - the ubiquitous plain old telephone system (POTS) on the one hand and the rapidly developing packet-data Internet on the other hand.

Personal Cordless Telephony (PCT) $/ 13 /$ is a service that Siemens currently implements for one of its international customers with the Service Node architecture. The service allows the access to a distinct subscriber from a group of persons via mobile telephony while calling a known fixed network number.

This service is the first step towards a complete PSTN, ISDN, DECT and GSM integration. In this manner, PCT is a basic service towards PCS that will build up the skeleton of much more complex communications services, such as routing of multimedia data throughout diverse networks.

The Personal Cordless Telephony and the Unified Media Communication Service (UMCS) presented in this paper build up together the base for the next generation telecommunications services. Whereas the PCT service introduces Distributed Intelligent Network Elements (DINE), Service Nodes, in the IN routing techniques of the PCS world, the @INGate architecture and the UMC service provide a practical approach to Enhanced Media Contents Provisioning (EMCP) in the CTNI area. 
Both services are mutually complementary and build the ground pillars of a layered service model (LSM) in an integrated media communications architecture (IMCA). These two basic services are enhanced with additional components and functionalities spread out throughout all media and network types in a similar way that the layers of an OSI protocol stack implement particular characteristics of the communication process.

Whereas the first pillar of services will grow horizontally, thus including new network types and routing mechanisms, the second one will grow vertically in order to develop complex applications with different (possibly hierarchically structured) media types such as video-telephony, conferencing, teleworking, teleoffice, telefactory, virtual enterprise, etc.)

Both services will be developed, justified and tested in an interplay relation in order to be finally merged in a configurable CTNI service. The evolution of the Network-Bridge Service Node is characterized by the following features:

-NB-SN as multimedia platform represents a mirroring (extension) of the data networks concerning news and data transfer, as well as tele-(co)operation services;

-NB-SN as network bride (CTNI) allows the access to other networks, resources and services: radio and satellite networks, electronical and optical broadband networks, etc.;

-NB-SN as a glue element (mediation function) in access networks and thus to closed loop interactive services in the business and entertainment areas;

-NB-SN as first step towards the realisation of true active intellient networks and thus towards the complete integration of autonomous data, programs, services and functionalities (mindware) within the global telecommunications network of all physical carriers.

Regarding the new developments in the packet network technology such as seamless ATM networks, new flexible addressing types, intelligent switches, agent routing and management methods, it is marked out that the network bridge service node will obtain the same characteristics and functionalities in order to guarantee its mediation function among the communications networks.

There is one distict trend that can be clearly discerned: the Distributed Intelligent Network Element (DINE) Service Node will continue to grow.

\section{Acknowledgements}

The work discussed in this paper was performed in the context of a project grant by Siemens AG. We would like to acknowledge the support of Prof. Carl, director of IN development at Siemens AG - Berlin who initiated the @INGate project. Many thanks to Hewlett Packard for the donation of the HP OpenView management platform as part of their HPOUA activities [12].

\section{References}

[1] P. L. Simeonov, MediaWeb - Product Definition, Doc. Nr. P30308-A7957-A000-04-7618, Siemens AG, OEN TI PSI1, 1996.

[2] P. L. Simeonov, Add-On IN Gateway Services for Service Node in Distributed Telecom Networks, Internal Paper, Siemens AG, Oct. 1996.

[3] Peter Bocker; ISDN - Digitale Netze für Sprach-, Text-, Daten-, Video- und Multimediakommunikation; Springer; 1997; ISBN 3540-57431-X

[4] Alan O. Freier and Philip Karlton and Paul C. Kocher, The SSL Protocol Version 3.0: ftp:// ftp.iesd.auc.dk/pub/ietf/internet-drafts/draftfreier-ssl-version3-01.txt

[5] ITU-T, ITU-T Recommendation Series X.7xx Data Communication Networks Management

[6] ITU-T, ITU-T Recommendation Series M.3xxx Maintenance Telecommunications Management Network

[7] ETSI, ETR 067, „Baseline Document on the Integration of IN and TMN", Sept. 1992

[8] DigiCash; DigiCash ecash - cybershops home page; http://www.digicash.com/shops/cybershop.html

[9] Mark Twain Bank; Alphabetical list - Mark Twain Bank Ecash Accepting Shops; http:// www.marktwain.com/shops.html

[10] Mastercard; Secure Electronic Transactions; http://www.mastercard.com/set/; 1997

[11] Daniel C. Lynch and Leslie Lundquist, digital money, John Wiley \& Sons, Inc, NY, ISBN 0471-14178-X.

[12] HP OUA; Homepage; http://www.ovoua.org

[13] Personal Cordless Telephony: Description, Siemens A, OEN TI, Doc. Nr. P30308-A7671T000-01-7618, 18.06.1996, A. Vogel 